\title{
Criteri editoriali
}

I Commentaria et Lexica Graeca in Papyris reperta (CLGP) sono divisi in quattro parti:

I) Commentaria et Lexica in auctores. Testi papiracei che contengono testimonianze dell'esegesi ad autori identificati. Tali testi possono appartenere alle seguenti tipologie: hypomnemata; hypotheseis; syngrammata; glossari e lessici a singoli autori; voci di lessici riportabili a un autore; marginalia (scholia e glosse). Sono stati tralasciati i papiri che presentano segni marginali e varianti senza che si riscontri alcun commento e quelli di contenuto esclusivamente biografico, mentre sono inclusi i testi comprensivi di elementi sia esegetici che biografici. A discrezione del curatore, inoltre, potranno essere considerati altri materiali di carattere esegetico.

La parte I sarà costituita da quattro volumi, a loro volta suddivisi in fascicoli:

1. Aeschines-Bacchylides

2. Callimachus-Hipponax

3. Homerus

4. Hyperides-Xenophon

II) Commentaria in adespota. Testi esegetici riferiti a opere e autori non identificati, raggruppati secondo il genere letterario del testo commentato (epica, lirica etc.).

III) Lexica. Prodotti di carattere lessicografico generale (i glossari e i lessici a singoli autori rientrano nella parte I; non sono compresi i lessici bilingui). Si osservi che i termini 'glossario' e 'lessico' non sono usati come sinonimi: il glossario presenta i lemmi nell' ordine in cui compaiono in una determinata opera di un autore; nei lessici, invece, i lemmi seguono l'ordine alfabetico e possono essere tratti da autori e opere diversi.

IV) Concordantiae et Indices. Un articolato sistema di riferimenti incrociati permetterà il reperimento dei materiali secondo diverse "chiavi" di accesso (per es. le citazioni degli autori e dei grammatici).

In generale nel CLGP i papiri sono disposti per autori commentati, in ordine alfabetico secondo la forma latina del nome. Per ogni autore si prevede un'introduzione generale, quindi l'esame dei papiri che conservano materiali esegetici relativi alle opere, presentate in ordine alfabetico secondo la forma 
latina del titolo (quelle non identificate si trovano in fondo); quando questo criterio non risulta applicabile i testi seguono l'ordine alfabetico per collezione papirologica.

Un punto interrogativo dopo il numero assegnato a un determinato papiro contraddistingue, di norma, $i$ reperti attribuiti a un autore in forma dubitativa. Se nessuna attribuzione risulta accettata dal curatore, il papiro sarà pubblicato fra i Commentaria in adespota. Se il carattere esegetico dell'opera non è sicuro, il punto interrogativo seguirà il titolo che identifica il genere dell'opera. Non sono numerati autonomamente, ma solo descritti in brevi schede, individuate da una lettera, i frammenti sulla cui natura permangono incertezze radicali.

Le sigle dei papiri sono tratte dalla Checklist of Editions of Greek, Latin, Demotic and Coptic Papyri, Ostraca and Tablets, Ed. J.F. Oates - R.S. Bagnall - S.J. Clackson - A.A. O'Brien - J.D. Sosin - T.G. Wilfong - K.A. Worp, BASP Suppl. $9,2001^{5}$. Una versione continuamente aggiornata si trova on line all'indirizzo: http:/ / papyri.info/ docs/ checklist.

Le riviste sono abbreviate secondo le sigle de L'Année Philologique. Bibliographie critique et analytique de l'antiquité gréco-latine, Paris 1928-.

Per i nomi e le opere degli autori greci si utilizzano le abbreviazioni del Vocabolario della lingua greca di Franco Montanari (= GI, 2013³), pp. 15-63: in caso di autori omonimi ivi diversificati con esponente o di opere indicate con un numero, si è ricorso ad abbreviazioni perspicue, confrontando il LSJ ed eventualmente il Thesaurus Linguae Graecae. Canon of Greek Authors and Works, by L. Berkowitz-K.A. Squitier, New York-Oxford $1990^{3}$ (versione on line aggiornata al sito http:/ / stephanus.tlg.uci.edu). Per le opere e gli scrittori latini si segue 1'Oxford Latin Dictionary, Ed. by P.G.W. Glare, Oxford $2012^{2}$ (versione on line aggiornata al sito www.oxfordscholarlyeditions.com/page/abbreviations).

All'inizio di ogni scheda si forniscono una serie di indicazioni così suddivise:

Prov.: Provenit (luogo di ritrovamento, secondo la denominazione latina).

Cons.: Conservatur (luogo di conservazione).

Ed./Edd.: Edidit/Ediderunt (edizioni del testo; le abbreviazioni bibliografiche che compaiono in questa sezione possono trovarsi anche sotto la voce Comm.).

Tab./Tabb.: Tabula/Tabulae (indicazioni delle immagini esistenti).

Comm.: Commentationes (numerazione in $\mathrm{MP}^{3} \mathrm{e}$ in $\mathrm{Pack}^{2}$, se differente; quindi il numero di $\mathrm{LDAB}$. Le sigle $\mathrm{MP}^{3} \mathrm{e} \mathrm{LDAB}$ rimandano ai repertori disponibili on line, rispettivamente agli indirizzi:

web.philo.ulg.ac.be/cedopal/ base-de-donnees-mp3 www.trismegistos.org/ldab. 
Segue la bibliografia in ordine cronologico e in forma abbreviata: le indicazioni bibliografiche complete si trovano nel Conspectus librorum).

Per ciò che concerne i commentari, dopo un'introduzione sul papiro, si offre la trascrizione letteraria dell'intero testo con i lemmi in grassetto. Il grassetto è usato anche per contraddistinguere i lemmi nelle voci di lessico e nelle annotazioni marginali. Riguardo a queste ultime, si valuta caso per caso se fornire la trascrizione sia del testo letterario (a volte solo parziale), sia delle note, riproducendo fedelmente la posizione dei marginalia, oppure se indicare unicamente il lemma a cui le note marginali stesse si riferiscono.

Se nel papiro vi è iota mutum, nell'edizione del testo viene ascritto; in caso contrario, nel testo è sottoscritto. Le scritture anomale sul papiro sono riportate in apparato papirologico e normalizzate nel testo secondo gli usi correnti (ad

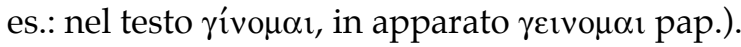

Sono usati i numeri romani per le colonne, i numeri arabi per i righi. Si adotta la numerazione dei righi per colonne, anche quando nell'edizione di riferimento compare la numerazione continua.

La traduzione, se presente (vi sono casi in cui il curatore non ha ritenuto opportuno inserirla), è posta generalmente dopo gli apparati; a discrezione del curatore può trovarsi anche nelle note di commento.

Le diverse lingue in cui potranno essere scritti i contributi inevitabilmente determineranno alcune difformità e/o necessari adattamenti redazionali.

Per la citazione del CLGP si utilizzerà il seguente criterio: il nome dell'autore, accompagnato dal numero che contrassegna il papiro, quindi la sigla della raccolta (ad es. Aeschylus 1 CLGP). Per i rimandi interni si usa il simbolo di una freccia $(\Rightarrow)$ in unione alle indicazioni della parte (in numero romano: $\Rightarrow$ III, a significare CLGP III Lexica) o del nome dell'autore, cui si aggiunge il numero identificativo del papiro (ad es. $\Rightarrow$ Aeschylus 1 ). All'interno della sezione su un autore, il richiamo a un papiro della stessa sezione è realizzato con il solo simbolo $\Rightarrow$ seguito dal numero. 